\title{
A INCLUSÃO DO BRINCAR NA HOSPITALIZAÇÃO INFANTIL
}

\author{
PLAYING GAMES WITH CHILDREN IN HOSPITAL
}

\author{
Maria Rita Zoéga SOARES ${ }^{1}$ \\ Universidade Estadual de Londrina \\ Dra. Maria Aparecida Trevisan ZAMBERLAN \\ Universidade Estadual de Londrina
}

\begin{abstract}
RESUMO
O presente artigo pretende discutir a importância da inclusão da atividade lúdica no atendimento à criança hospitalizada, bem como explicitar a utilização de tal recurso pelo psicólogo, equipe hospitalar e família do paciente. Considera-se o brincar uma atividade que pode facilitar o trabalho dos profissionais de saúde, porque propicia melhor adaptação e cooperação do paciente aos procedimentos médicos. Conclui-se que atividades lúdicas devem ser organizadas no hospital, por auxiliarem no desenvolvimento integral da criança, incrementando seu repertório comportamental.
\end{abstract}

\begin{abstract}
This article investigates the importance of ludical activities with children in hospital. It also demonstrates how it is used by the psychologist, the hospital staff and the patient's family. Playing is considered an activity which helps the work of the professionals of health because it leads to the patient better adaptation and cooperation towards medical procedures. Therefore, ludical activities should be offered in the hospitals because they help overall children's development improving their behavioural repertoire.
\end{abstract}

A literatura demonstra que a hospitalização tem um impacto sobre o comportamento da criança, levando à manifestação de reações adversas como o

(1) Docente do Departamento de Psicologia Geral e Análise do Comportamento na Universidade Estadual de Londrina Mestre em Educação (UEL) e Doutoranda em Psicologia Escolar e do Desenvolvimento Humano - USP (São Paulo). Autor para correspondência: R. Prefeito Hugo Cabral 1062, apto. 142 - Londrina - PR - CEP 86020917

Endereço para correspondência com o Editor: Universidade Estadual de Londrina Campus Universitário - Centro de Ciências Biológicas - Departamento de Psicologia Geral e Análise do Comportamento.

Caixa Postal: 6001 - CEP 86051990 - Londrina - PR - Fone: (043) 371-4227 - FAX: (043) 371-4207. 
estresse, a ansiedade e o medo (Azevedo, 1999; Costa Jr., 1999; Guimarães, 1999; Huerta, 1996; Jasinski, 2000; Miyazaki, Amaral \& Salomão, 1999; Santos, 2000).

Tais reações podem ser atribuídas a fatores relacionados à ausência de atividades cotidianas e escolares; ao trauma da doença; ao ambiente desconhecido; à distância de seu ambiente familiar e de seus pares; à incerteza a respeito de como se comportar adequadamente; à perda de controle e de autonomia; à ausência de rotina e aos procedimentos médicos e cirúrgicos (Sperb, Mello e Goulart, 1995; Azevedo, 1999; Lipp, 2000).

Intervenções no contexto hospitalar devem ser planejadas visando a promoção de condições favoráveis à reabilitação de efeitos de experiências adversas ao desenvolvimento comportamental da criança. O atendimento não deve se restringir ao individual de cada paciente, mas a todo contexto de internação, desde a estrutura ambiental, até a assistência em áreas que correspondem às suas necessidades. O trabalho deve considerar aspectos psicológicos, pedagógicos e sociológicos da criança e de sua família.

Quando pessoas experenciam situações como doença e hospitalização, a qualidade do ambiente pode afetar diretamente no processo de recuperação. Hospitais pediátricos deveriam investir na melhoria de oportunidades para cada indivíduo assistido, elaborando alternativas de organização em enfermarias pediátricas superpopulosas, no sentido de torná-las mais adequadas ao serviço prestado às crianças (Ranna, Jacob, Sakata, Valente, Ferrari, 1992).

A forma de organizar uma unidade de internação infantil deve considerar a estruturação de um ambiente que atenda mais adequadamente às necessidades do paciente. O brincar é uma atividade que deve ser inserida neste contexto, por facilitar a aprendizagem da criança, incentivando sua adaptação e cooperação.
O ambiente tem que enfatizar a saúde através de atividades, liberdade de escolha, mobilidade e espaço. Deve-se esperar que a criança desenvolva capacidade de interagir, aproveitando ao máximo as oportunidades para experenciar o brinquedo, passando de sujeito passivo a instigador e controlador ativo (Wishon \& Brown, 1991).

\section{O brincar para a criança hospitalizada}

A criança, mesmo hospitalizada, deve ter preservado seu direito ao brincar, porque o brinquedo é considerado um veículo para o desenvolvimento social, emocional e intelectual (Bomtempo, Hussein, \& Zamberlan, 1986). O recurso lúdico, neste contexto, não deve ser apenas um incentivo à diversão e entretenimento (Ribeiro, 1991), mas a uma alternativa educacional (quando empregado como incentivo ao desenvolvimento) e terapêutica (quando auxilia no trabalho para diminuir o estresse, o medo e a ansiedade).

Silva Jr. (2000) descreveu que a aprendizagem é mais eficaz quando é divertida. Assim, a aprendizagem de conceitos relacionados à doença e hospitalização pode ser mais facilmente trabalhada com a inclusão da atividade lúdica no hospital.

Azevedo (1999) considerou o brincar como uma das estratégias facilitadoras da adaptação da criança à condição de hospitalização, permitindo que a mesma expresse seus sentimentos, proporcionando melhora no repertório de enfrentamento desta condição. Crianças aprendem, através do brincar, acerca de seu mundo e de como lidar com esse ambiente (de objetos, tempo, espaço, estrutura e pessoas).

A utilização de técnicas lúdicas durante a hospitalização de crianças é uma estratégia efetiva para diminuir o estresse, o medo e a ansiedade relacionados com tal condição. Quando a criança encontra no hospital um espaço dedicado ao "brincar", pode ficar mais relaxada, pais menos ansiosos e ambos podem 
perceber que naquele local há uma preocupação com o bem-estar do indivíduo.

Para Sikilero, Morselli e Duarte (1997), o brincar deve ser explorado porque é uma das formas de expressão mais genuínas da criança. Uma vez que a linguagem verbal ainda não está suficientemente desenvolvida para expressão de sentimentos e pensamentos, tal recurso pode ser utilizado para facilitar a compreensão do comportamento deste paciente.

A participação da criança hospitalizada em atividades que envolvem o brincar, também pode acelerar sua recuperação, contribuindo para a diminuição do tempo de permanência no hospital e, conseqüentemente, o custo da hospitalização (Gottfried \& Brown, 1986).

Brincadeiras devem proporcionar diversão e produzir relaxamento; ajudar a criança a sentir-se mais segura e a diminuir o estresse por estar longe de casa; fornecer um meio para a expressão de sentimentos, para a estimulação, aprimoramento do desenvolvimento e das relações. Através de brincadeiras, crianças praticam como lidar com o complicado e estressante processo de viver, de se comunicar e de estabelecer relacionamentos satisfatórios com outras pessoas. (Whaley \& Wong, 1989).

\section{A psicologia no contexto hospitalar}

Atualmente, faz-se necessário o desenvolvimento de tecnologia comportamental adequada à situação hospitalar e à inclusão do psicólogo na equipe de saúde, para que o trabalho de prevenção e assistência à criança possa ser realizado (Guimarães, 1988).

O psicólogo tem sido solicitado para atuar no campo das relações entre pacientes e profissionais da saúde, da interdisciplinaridade, que caracteriza esse trabalho, auxiliando na compreensão de aspectos emocionais envolvidos na equipe, enfocando questões individuais e institucionais que possam interferir na qualidade assistencial (Ceccim \& Carvalho, 1997).

A intervenção psicológica deve também incluir a redução de ansiedade, buscando enfocar aspectos emocionais envolvidos na situação (como a promoção de auto-estima e de autoconfiança), além de aspectos relacionados ao vínculo entre pais e filhos (Wishon \& Brown, 1991).

O incentivo à expressão de sentimentos e fantasias também possibilita que a criança desenvolva um repertório para enfrentar a ansiedade, o medo de determinadas situações e a sensação de falta de controle. Como recurso terapêutico, o brinquedo pode ser utilizado como uma alternativa possível de expressão da criança no hospital.

A intervenção da psicologia em instituições hospitalares deve ir além dos objetivos prescritivos de facilitador do processo de adaptação da criança e da família à situação de hospitalização. Deve ser dirigido ao desenvolvimento de recursos para aprimorar a adaptação à hospitalização, incluindo no trabalho o motivo da internação da criança; a permissão da permanência da mãe junto a ela; a participação dos pais nos cuidados; a preparação psicológica para todos os procedimentos e, sobretudo, a utilização do brinquedo como parte integrante do ambiente hospitalar.

O psicólogo pode auxiliar crianças lidarem com aspectos negativos da hospitalização através do incentivo ao brincar. Nessa condição, tal atividade deve servir como proposta para permitir a compreensão de um ambiente não familiar além de lidar com conteúdos emocionais. O desenvolvimento de pesquisas relacionadas a condições que afetam o desenvolvimento da criança no contexto hospitalar, também pode auxiliar o psicólogo na prevenção e intervenção no contexto da saúde (Roberts \& McNeal, 1995; Azevedo, 1999). 


\section{Atuação do profissional da saúde}

O hospital deve apresentar características de acolhimento, demonstrando que pode funcionar a partir de uma noção muito ampla de ser humano, buscando considerar sentimentos nas relações profissionais com os pacientes. Questões de ordem orgânica, afetivas e sociais estão presentes nesse contexto e a saúde precisa ser considerada um tema de natureza interdisciplinar.

A integração entre os membros da equipe hospitalar favorece a criança perceber uma uniformidade de ações, aspecto fundamental na prevenção de desajustes emocionais que podem vir a manifestarem-se durante e/ou após uma experiência hospitalar. A equipe hospitalar deve agir de modo integrado, buscando continuidade e consistência. O atendimento pressupõe uma constante troca de informações entre os componentes, para que decisões, condutas e intervenções sejam definidas nesse âmbito (Ceccim \& Carvalho, 1997; Duarte, Muller, Bruni, Duarte, 1987; Ranna et al., 1992).

Para conseguir o envolvimento da equipe com objetivos definidos e integrados, aspectos na formação profissional em diversas áreas devem ser reavaliados, capacitando-os para um atendimento global ao paciente pediátrico. Tanto a prática com crianças hospitalizadas e as concepções da equipe a respeito do brincar neste contexto, como o conhecimento acadêmico a respeito do assunto, podem influenciar na maneira como a situação é tratada em cada unidade de internação infantil. Com isso, condição contribui para assegurar uma condição mais humana àquela população à formação de profissionais da saúde com uma nova concepção de assistência.

O brinquedo (ou o brincar) também pode servir como um elo na relação criança e profissional. A preocupação não deve enfocar apenas o tipo de atividade a ser desenvolvida, mas a relação que se estabelece entre as pessoas envolvidas (criança, pais e profissional), buscando trabalhar aspectos problemáticos relacionados à adaptação a doença e ao hospital.

O uso do brinquedo na assistência à criança hospitalizada pode ser benéfico tanto para a criança como ao profissional. O trabalho do psicólogo deve ser o de incentivo para que o profissional da saúde compreenda o que está sendo manifestado pela criança, respondendo contingentemente a estas manifestações. Tal condição, pode promover sentimentos positivos e de relaxamento para o paciente e equipe hospitalar (Azevedo, 1999).

O brincar deve auxiliar o profissional a compreender melhor as necessidades da criança porque facilita uma comunicação mais efetiva com ela. O paciente pode reconhecer, através do brincar com o profissional, a capacidade de que ele possa ser seu intérprete e defensor, uma pessoa adulta em que ela possa reconhecer como aliado e possa confiar (Lindquist, 1993).

\section{- enfoque na família da criança hospitalizada}

A percepção dos pais e das crianças sobre o empenho e integração da equipe hospitalar influencia de uma forma direta no relacionamento equipe-família, condição indispensável para o processo de cura. No hospital, pais também podem se sentir mais seguros se souberem que as necessidades emocionais de seus filhos estão sendo alvo de intervenções, assim como as necessidades médicas (Wishon e Brown, 1991).

O papel da família tem sido historicamente reconhecido como um elemento essencial para a saúde psíquica e física da criança no contexto hospitalar. Pais devem participar de uma forma ativa, restaurando seu papel como agentes cuidadores e restabelecendo maior confiança em sua capacidade pessoal para lidar com o filho.

A preparação sistemática da criança e de seus pais com relação a hospitalização 
através de atividades lúdicas, pode levar a uma redução de inadequações comportamentais, alterações emocionais, melhor adaptação à condição e um maior ajustamento social depois da alta.

A prática tem demonstrado que o brinquedo também pode auxiliar pais a perceberem seus filhos com maior potencial e capacidade, influenciando diretamente na autoconfiança dessas crianças. Pais devem ter oportunidade de observarem o filho desempenhando atividades lúdicas, podendo verificar que a criança é capaz de desenvolver repertório alternativo para se adaptar àquela condição (Azevedo, 1999).

O ambiente deve ser considerado terapêutico também para pais, quando observam o filho brincando e interagindo com outras crianças, apesar da enfermidade. Deve haver um empenho para a valorização da díade criança-família, evitando esta dissociação na experiência de hospitalização.

\section{CONCLUSÃO}

O presente artigo se propôs a discutir, dentro de uma visão mais abrangente do conceito de saúde, aspectos relacionados à utilização do brincar na hospitalização infantil, à inclusão do trabalho do psicólogo neste contexto, à atuação do profissional da saúde e a condição da família do paciente.

Cabe a Psicologia auxiliar o trabalho da equipe hospitalar, investindo na recuperação física e emocional da criança. Atividades que incluam brincadeiras devem ser organizadas no hospital, por auxiliarem no desenvolvimento da criança, incrementando seu repertório comportamental através da experimentação de diferentes respostas de ajustamento ao meio.

Através do lúdico, pode-se explorar a criatividade e a capacidade de improvisação do profissional da saúde, para poder suprir eventuais dificuldades (falta de espaço, de material e de pessoal) e transformar o brinquedo em possibilidades ilimitadas. A atual condição dos hospitais brasileiros exige uma adaptação de estratégias de profissionais para que possam desenvolver trabalhos efetivos.

O profissional que trabalha no contexto da saúde deve se utilizar de pesquisas recentes relacionadas ao desenvolvimento humano, à comunicação com crianças, à utilização do lúdico e à aplicação de determinados princípios para sua prática, assegurando que crianças sejam ouvidas e compreendidas.

Neste sentido, deve-se levar em conta a busca de uma melhor qualidade de vida dentro da instituição de saúde, considerando a integridade do indivíduo em toda sua especificidade, incluindo a recuperação do organismo doente e de suas necessidades.

\section{REFERÊNCIAS BIBLIOGRÁFICAS}

AZEVEDO, M. R. Z. S. (1999). Papel e Importância do Lúdico para Profissionais da Saúde: Análise de Jogos e Brincadeiras no Contexto Hospitalar. Dissertação de Mestrado, Departamento de Educação, Universidade Estadual de Londrina, $\mathrm{Pr}$.

BOMTEMPO, E., Hussein, C. L., \& Zamberlan, M. A. T. (1986). Psicologia do Brinquedo: Aspectos teóricos e metodológicos. São Paulo: Nova Stella.

CECCIM, R. B., \& Carvalho, P. R. A. (1997). Criança Hospitalizada: atenção integral como escuta à vida. Porto Alegre: UFRGS.

COSTA Jr., Á. L. (1999). Psicooncologia e Manejo de Procedimentos Invasivos em Oncologia Pediátrica; Uma Revisão de Literatura. Psicologia: Reflexão e Crítica, 12(1), 107-118.

DUARTE, E. R. M., Muller, A. M., Bruno, S. M. A., \& Duarte, A. L. S. (1987). A utilização do brinquedo na sala de recuperação: um recurso a mais para a assistência de 
enfermagem à criança. Revista Brasileira de Enfermagem, 40(1), 74-81.

GotTFRIED, A. W., \& Brown, C. C. (1986). Play interactions: The contribution of play material and parental involvement in children's development. Lexington: Lexington Books.

GUIMARÃES, S. S. (1988). A Hospitalização na Infância. Psicologia: Teoria e Pesquisa, 4(2), 102-112.

GUIMARÃES, S. S. (1999). Psicologia da Saúde e doenças crônicas. In R. R. Kerbauy (Org.), Comportamento e Saúde: explorando alternativas (pp.22-45). Santo André, SP: ARBytes.

HUERTA, E. P. N. (1996). Preparo da criança e família para procedimentos cirúrgicos: Intervenção de enfermagem. Revista da Escola de Enfermagem da USP, 30(2), 340-53.

JASINSKI, D. M. (2000). Use of play therapy in same day pediatric surgery; Impact on patient and parent anxiety and level of parent satisfaction. The Catolic University of America, Degree DNSc.

LINDQUIST, I. (1993). A criança no hospital: terapia pelo brinquedo. São Paulo: Scritta.

LIPP, M. E. N. (2000). Crianças estressadas: Causas, sintomas e soluções. Campinas: Papirus.

MIYASAKI, M. C. O.; Amaral, V. L. A. R.; Salomão, J. B. (1999). Asma na infância: dificuldades no manejo da doença e problemas comportamentais. In R. R. Kerbauy (Org.), Comportamento e Saúde: explorando alternativas (pp.99-118). Santo André, SP: ARBytes.

RANNA, W., Jacob, C. M. A., Sakata, C. K., Valente, M. T., Ferrari, V. P. M. (1992). Atuação da Equipe Multidisciplinar em Enfermaria Pediátrica com pais participantes. Revisões e Ensaios.
RIBEIRO, C. A. R. (1991). O efeito da utilização do brinquedo terapêutico pela enfermeira pediatra, sobre o comportamento de crianças recém-hospitalizadas. Revista da Escola de Enfermagem, 25(1), 41-60.

ROBERTS, M. C., \& McNeal, R. E. (1995). Historical and Conceptual Foundations of Pediatric Psychology. In M. C. Roberts (Ed.), Handbook of Pediatric Psychology (pp. 3-18). New York: Guilford.

SANTOS, A. R. R. (2000). Psicooncologia pediátrica em um Hospital-Escola. In R. R. Kerbauy (Org.), Sobre Comportamento e Cognição: Conceitos, pesquisa e aplicação, a ênfase no ensinar, na emoção e no questionamento clínico (pp. 139-147). Santo André, SP: SET.

SILVA Jr., S. C. (2000). Fisiologia e Manejo da dor. In R. R. Kerbauy (Org.), Sobre Comportamento e Cognição: Conceitos, pesquisa e aplicação, a ênfase no ensinar, na emoção e no questionamento clínico (pp.133-138). Santo André, SP: SET.

SIKILERO, R. Morselli, R., \& Duarte, G. (1997). Recreação: uma proposta terapêutica. Em R. Ceccim \& P. Carvalho (Orgs.), Criança Hospitalizada: atenção integral como escuta à vida (pp. 59-65). Porto Alegre: Editora da Universidade.

SPERB, T. M., Mello, C. O., Goulart, C., \& Ew, R. (1995). Brincar no Hospital: O que pensa e como age a equipe de profissionais de internação infantil de Porto Alegre. Projeto de Pesquisa, Porto Alegre: Instituto de Filosofia e Ciências Humanas.

WHALEY, L. F., \& Wong, D. L. (1989). Enfermagem Pediátrica: Elementos Essenciais à Intervenção Efetiva. Rio de Janeiro: Guanabara.

WISHON, P. M., \& Brown, M. H. (1991). Play and the young hospitalized patient. Early Child Development and Care, 72, 39-46. 